\title{
Habilitação em terapia floral para cirurgiões-dentistas: uma análise por estados e regiões brasileiras
}

\author{
Habilitation in floral therapy for dentists: an analysis by Brazilian states and regions \\ Habilitación en terapia floral para dentistas: un análisis por estados y regiones brasileñas \\ José de Alencar FERNANDES NETO ${ }^{1}$ \\ Thamyres Maria Silva SIMÕES ${ }^{1}$ \\ Jhonatan Thiago LACERDA-SANTOS ${ }^{2}$ \\ Allyson Martim Medeiros LIRA ${ }^{3}$ \\ Maria Helena Chaves de Vasconcelos CATÃO
}

\begin{abstract}
Doutorando(a), Programa de Pós-Graduação em Odontologia, Departamento de Odontologia, Universidade Estadual da Paraíba, UEPB 58429-500, Campina Grande-PB, Brasil ${ }_{2}^{2}$ Mestrando Programa de Pós-Graduação em Odontologia, Departamento de Odontologia, Universidade Estadual da Paraíba, UEPB 58429-500, Campina Grande-PB, Brasil ${ }^{3}$ Graduando em Odontologia, Departamento de Odontologia, Universidade Estadual da Paraíba, UEPB 58429-500, Campina Grande-PB, Brasil ${ }^{4}$ Professora Doutora do Programa de Pós-Graduação em Odontologia, Universidade Estadual da Paraíba, UEPB 58429-500, Campina Grande-PB, Brasil
\end{abstract}

\section{Resumo}

Introdução: A terapia floral faz parte de um campo emergente de terapias complementares, caracterizado pelo uso de meios menos onerosos, fundado numa visão integral da saúde e, principalmente, por métodos não invasivos e não tóxicos. No Brasil, esta terapia vem ganhando espaço, principalmente a partir da aprovação da Política Nacional de Práticas Integrativas Complementares (PNPIC), que possibilita a articulação entre os sistemas médicos complexos e a medicina tradicional e complementar, visando à prevenção de doenças, recuperação, promoção e racionalização das ações em saúde. Objetivo: avaliar a quantidade de cirurgiões-dentistas habilitados em terapia floral e número de cursos já oferecidos dessa habilitação por estados e regiões do Brasil. Material e Método: Os dados referentes aos números de cirurgiõesdentistas habilitados e a quantidade de cursos de habilitação em terapia floral foram coletados diretamente do site do Conselho Federal de Odontologia (CFO), assim como a distribuição geográfica desses no Brasil. Todos os dados utilizados nesta pesquisa são de acesso público. Resultados: São Paulo detém o maior número de cirurgiões-dentistas habilitados em terapia floral (n=16) e é o estado brasileiro, onde o único curso de habilitação foi ministrado. Por regiões, o Sudeste apresentou o maior número de profissionais habilitados no país (56,8\%). No Sul, para cada habilitado existem 5.586,6 cirurgiões-dentistas, enquanto que no Nordeste este número cresce para 16.425. Conclusão: Há poucos cirurgiões-dentistas habilitados e cursos de habilitação em terapia floral em todo o país, tendo maior concentração na região Sudeste, principalmente no estado de São Paulo.

Descritores: Essências Florais; Terapias Complementares; Odontologia; Odontólogos.

\section{Abstract}

Introduction: Floral therapy is part of an emerging field of complementary therapies, characterized by the use of less expensive means, based on an integral view of health and, mainly, non-invasive and non-toxic methods. In Brazil, this therapy has been gaining ground, especially since the adoption of the National Policy on Complementary Integrative Practices (PNPIC), which allows the articulation between complex medical systems and traditional and complementary medicine, aiming at disease prevention, recovery, promotion and rationalization of health actions. Objective: to evaluate the number of dentists qualified in floral therapy and the number of courses already offered by this qualification by states and regions of Brazil. Material and methods: The data referring to the numbers of qualified dental surgeons and the number of qualification courses in floral therapy were collected directly from the website of the Federal Council of Dentistry (CFO), as well as the geographical distribution of these in Brazil. All data used in this survey are publicly accessible. Results: São Paulo has the largest number of dentists qualified in floral therapy $(\mathrm{n}=16)$ and is the Brazilian state, where the only qualification course was given. By region, the Southeast has the highest number of qualified professionals in the country (56.8\%). In the South, there are 5,586.6 dental surgeons for each qualified, while in the Northeast this number grows to 16,425. Conclusion: There are few qualified dental surgeons and habilitation courses in flower therapy throughout the country, with a higher concentration in the Southeast region, mainly in the state of São Paulo.

Descriptors: Flower Essences; Complementary Therapies; Dentistry; Dentists.

\section{Resumen}

Introducción: La terapia floral forma parte de un campo emergente de terapias complementarias, caracterizado por el uso de medios menos onerosos, fundado en una visión integral de la salud y, principalmente, por métodos no invasivos y no tóxicos. En Brasil, esta terapia viene ganando espacio, principalmente a partir de la aprobación de la Política Nacional de Prácticas Integrativas Complementarias (PNPIC), que posibilita la articulación entre los sistemas médicos complejos y la medicina tradicional y complementaria, buscando la prevención de enfermedades, recuperación, promoción y racionalización de las acciones en salud. Objetivo: evaluar la cantidad de dentistas habilitados en terapia floral y número de cursos ya ofrecidos de esa habilitación por estados y regiones de Brasil. Material y Método: Los datos referentes a los números de dentistas habilitados y la cantidad de cursos de habilitación en terapia floral fueron recolectados directamente del sitio del Consejo Federal de Odontología (CFO), así como la distribución geográfica de esos en Brasil. Todos los datos utilizados en esta investigación son de acceso público. Resultados: São Paulo posee el mayor número de dentistas habilitados en terapia floral ( $\mathrm{n}=16)$ y es el estado brasileño, donde el único curso de habilitación fue dictado. Por regiones, el Sudeste presentó el mayor número de profesionales habilitados en el país $(56,8 \%)$. En el Sur, para cada habilitado existen 5.586,6 dentistas, mientras que en el Nordeste esta cifra crece a 16.425 . Conclusión: Hay pocos dentistas habilitados y cursos de habilitación en terapia floral en todo el país, teniendo mayor concentración en la región Sudeste, principalmente en el estado de São Paulo.

Descriptores: Esencias Florales; Terapias Complementarias; Odontología; Odontólogos.

\section{INTRODUÇÃO}

A terapia floral, segundo a Resolução $\mathrm{n}^{\circ}$. 82/2008 do Conselho Federal de Odontologia (CFO) pode ser definida como "prática complementar ao bem-estar da saúde, na medida em que consiste no uso de essências florais como método de tratamento, focando a atenção no indivíduo e não na doença, podendo ser usada em qualquer pessoa, de todas as idades, não possuindo contraindicações e nem produzindo interações medicamentosas, oferecendo uma forma ampla de prevenção e humanização"1.

Essa terapia utiliza essências florais, que originalmente foram descobertas pelo médico inglês Edward Bach, que estudou a natureza curativa das flores. Essas essências agem sobre as energias negativas que desenvolvem desordens na saúde, recuperando o equilíbrio natural do organismo, tornando os usuários com menos propensão a doenças ${ }^{2}$. 
A terapia floral é regulamentada em vários países como, Austrália, Cuba, Estados Unidos, Reino Unido, Itália e Canadá ${ }^{1,3-5}$ e vem sendo utilizada em algumas áreas da saúde como a Enfermagem, Farmácia, Fisioterapia e Odontologia ${ }^{1}$, com diversas indicações, como por exemplo: controle da dor, ansiedade, medo e estresse $e^{2,3,6}$.

Em 2006, a Portaria $n^{\circ} .971$ instituiu a Política Nacional de Práticas Integrativas e Complementares no Sistema Único de $\mathrm{Saúde}^{7}$, e a partir de então, deu-se início a busca pela habilitação nestas práticas em Odontologia.

O Conselho Federal de Odontologia (CFO), através da Decisão CFO 45/2008, constituiu a habilitação de cirurgiões-dentistas em modalidades de Práticas Integrativas, Alternativas ou Complementares, dentre elas a terapia floral ${ }^{8}$.

Diante disso, este estudo tem por objetivo avaliar a quantidade de cirurgiões-dentistas habilitados em terapia floral e o número de cursos já oferecidos dessa habilitação por estados e regiões do Brasil.

\section{MATERIAL E MÉTODO}

Os dados referentes aos números totais de cirurgiões-dentistas e as informações sobre os cursos de habilitação já ministrados no país foram coletados diretamente do site do $\mathrm{CFO}^{9}$, assim como a distribuição geográfica desses de acordo com as unidades federativas brasileiras. $\mathrm{O}$ número total de profissionais habilitados em terapia floral por estado foi requisitado via $e$-mail ao CFO por um dos autores deste estudo. $\mathrm{Na}$ pesquisa, foram considerados apenas aqueles profissionais que estavam em atividade, de acordo com o registro no Conselho.

Quanto aos cursos de habilitação em terapia floral, foram recolhidas as seguintes informações também no site do $\mathrm{CFO}^{9}$ : local do curso, período, carga horária e número de vagas oferecidas.

A pesquisa foi realizada em julho de 2018, conseguindo-se assim o número exato de profissionais e dos cursos da habilitação oferecidos em cada estado, devidamente cadastrados no Conselho, até a data da busca. Todos os dados utilizados nesta pesquisa são de acesso público.

\section{RESULTADOS}

Em todo o Brasil, existem 37 cirurgiõesdentistas habilitados em terapia floral. Dentre os estados brasileiros, São Paulo detém o maior número desses profissionais $(n=16)$ (Tabela 1), e é o estado onde o único curso da habilitação foi ministrado entre os anos de 2010 e 2011, com 256 horas de carga horária (Tabela 2).

Por regiões, o Sudeste abriga $21(56,8 \%)$ dos profissionais habilitados no país e a região Norte do Brasil não apresenta cirurgiões-dentistas com a habilitação em questão. No Sul, a proporção habilitados/cirurgião-dentista é de 1/5.586,6
(Tabela 3).

Tabela 1. Distribuição do número de cursos da habilitação ministrados, de profissionais habilitados e relação habilitados/cirurgião-dentista por unidade federativa brasileira.

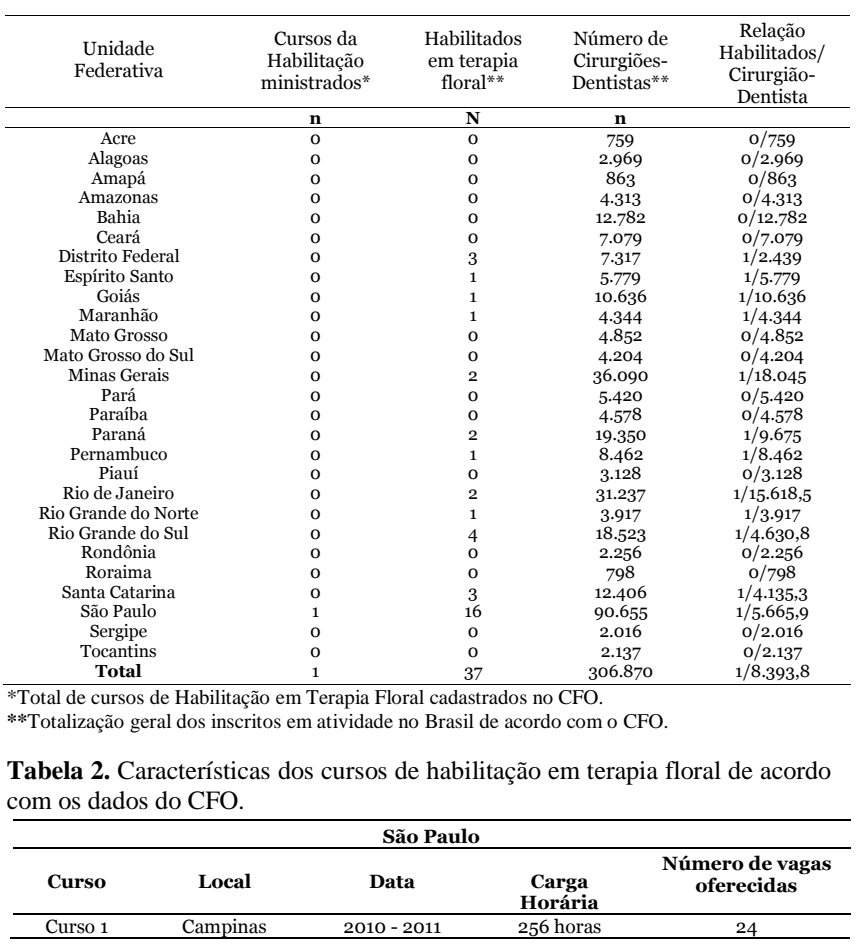

Tabela 3. Distribuição percentual de cursos da habilitação ministrados, de profissionais habilitados e relação habilitados/cirurgião-dentista por unidade federativa brasileira.

\begin{tabular}{|c|c|c|c|c|}
\hline $\begin{array}{c}\text { Região } \\
\text { brasileira }\end{array}$ & $\begin{array}{c}\text { Cursos de } \\
\text { Habilitacão } \\
\text { ministrados* }\end{array}$ & $\begin{array}{l}\text { Habilitados } \\
\text { em terapia } \\
\text { floral }^{* *}\end{array}$ & $\begin{array}{l}\text { Número de } \\
\text { Cirurgióes- } \\
\text { Dentistas*** }\end{array}$ & $\begin{array}{c}\text { Relacão } \\
\text { habilitados/ } \\
\text { Cirurgião-dentista }\end{array}$ \\
\hline & n (\%) & n (\%) & n (\%) & \\
\hline Centro-Oeste & $0(0 \%)$ & $4(10,8 \%)$ & $27.009(8,7 \%)$ & $1 / 6.752,3$ \\
\hline Nordeste & $\mathrm{o}(\mathrm{o} \%)$ & $3(8,1 \%)$ & $49.275(16,1 \%)$ & $1 / 16.425$ \\
\hline Norte & $\mathrm{o}(\mathrm{o} \%)$ & $0(0 \%)$ & $16.546(5,4 \%)$ & $0 / 16.546$ \\
\hline Sudeste & $1(100 \%)$ & $21(56,8 \%)$ & $163.761(53,4 \%)$ & $1 / 7.798,1$ \\
\hline & $0(0 \%)$ & $9(24,3 \%)$ & $50.279(16,4 \%)$ & $1 / 5.586,6$ \\
\hline Total & $1(100 \%)$ & $37(100 \%)$ & $306.870(100 \%)$ & $1 / 8.393,8$ \\
\hline
\end{tabular}

**Totalizaç̃o geral dos inscritos em atividade no Brasil de acordo com o CFO.

\section{DISCUSSÃO}

Em Odontologia, a terapia floral tem sido mais relatada no gerenciamento da ansiedade e do medo ao tratamento odontológico. A redução de efeitos adversos torna essa terapia uma ferramenta importante e o baixo custo somado ao fácil acesso permitem grandes possibilidades de tratamento complementar, ratificando a importância da capacitação do cirurgião-dentista ${ }^{10}$.

São atribuições do Terapeuta Floral em Odontologia: I - tratar uma determinada pessoa e uma condição particular; II - atuar sobre a origem das doenças do sistema estomatognático; III - ter uma visão integral do paciente aliada à ciência e tecnologia, focando a atenção no indivíduo e não na doença, oferecendo uma forma ampla de prevenção e humanização na prática odontológica; e, IV - atuar no estado emocional do paciente, facilitando a prática odontológica $^{1}$. Desta forma, o cirurgião-dentista se torna um promotor da saúde geral do paciente ampliando as possibilidades de um tratamento integralizado ${ }^{11}$.

Estudos verificaram a quantidade de cirurgiões-dentistas habilitados em outras terapias 
complementares, observando que há 593 em laserterapia $^{12}$ e 410 em acupuntura ${ }^{13}$. No presente estudo, verificou-se que esse dado é ainda menor para a Terapia Floral, apresentando apenas 37 profissionais registrados no CRO.

Cabe destacar também que alguns profissionais possam ter realizado o curso e não terem cadastrado a habilitação no CFO. Além disso, nos estados que apresentam profissionais habilitados, estes podem estar centralizados em apenas cidades de médio ou grande porte, gerando uma distribuição desigual.

Desde a regulamentação da atividade de Terapia floral o CFO vem trabalhando em função do credenciamento de instituições que ofereçam os cursos de habilitação cuja carga horária mínima é de 180 horas ${ }^{1}$. Entretanto, os resultados desta pesquisa constataram que no Brasil existe apenas um curso de habilitação em terapia floral promovido pela Associação de Cirurgiões Dentistas APCD/Campinas no estado de São Paulo.

A Resolução CFO-82/2008 ${ }^{1}$ determinou que o profissional que em até 180 dias da data da publicação da mesma, comprovou utilizar acupuntura, há cinco anos dentro dos últimos dez anos, pôde requerer a habilitação, após análise pelo CFO. Isso pode justificar tanto o número de habilitados em terapia floral $(n=37)$ ser maior que a quantidade de vagas totais oferecidas pelo único curso da habilitação registrado $(n=24)$, quanto a presença de habilitados em outros estados brasileiros.

É importante destacar que apenas um único estado brasileiro ofereceu o curso de habilitação em terapia floral e apesar disso, existem profissionais habilitados nas outras regiões do Brasil, com exceção do Norte. Uma das explicações possíveis para esse fato é a de que os cirurgiões-dentistas realizaram a habilitação em São Paulo e retornaram ou migraram de cidades, onde atuam profissionalmente.

A escassez de cursos em Terapia Floral pode ser justificada devido a pouca procura dos profissionais, principalmente por falta de conhecimento sobre essa terapia em Odontologia. Observa-se que o conhecimento dos estudantes de odontologia de Universidades públicas sobre as terapias integrativas e complementares é baixo, tendo em vista que as grades curriculares das faculdades de odontologia no Brasil não contemplam o ensino dessas práticas ${ }^{14}$. Portanto, uma forma de difundir essa técnica, seria a inserção de disciplinas na graduação em Odontologia.

Contudo, observa-se a necessidade de ofertar novos cursos de habilitação em Terapia Floral para capacitar mais profissionais em todo o Brasil, preferencialmente em regiões estratégicas, a fim de descentralizar o conhecimento que atualmente se concentra na região Sudeste. Desta forma, ampliaria o acesso a essa terapia e consequentemente possibilita o benefício da terapia floral aos pacientes.

\section{CONCLUSÃO}

Conclui-se que há uma pequena quantidade de cirurgiões-dentistas habilitados em terapia floral em todo o Brasil, tendo maior concentração na região Sudeste. Proporcionar a abertura de novos cursos de habilitação em terapia floral, sobretudo nas regiões que nunca os disponibilizaram, poderia difundir essa técnica e viabilizar a habilitação de novos profissionais, proporcionando aos pacientes o acesso a esse tratamento.

\section{REFERÊNCIAS}

1. CFO. Conselho Federal de Odontologia. Resolução $n^{\circ} 82$ de 25 de setembro de 2008. DOU 190 de 01/10/2008.

2. Salles LF, Silva MJP. Efeito das essências florais em indivíduos ansiosos. Acta Paul Enferm. 2012;25(2):238-42.

3. Balinski AA. Use of Western Australian flower essences in the management of pain and stress in the hospital setting. Complement Ther Nurs Midwifery. 1998;4(4):111-17.

4. Rodríguez-Martín BC, Fallas-Durán M, Gaitskell B, Vega-Rojas D, Martínez-Chaigneau P. Predictors of positive opinion about Bach Flower Remedies in adults from three Latin-American countries: An exploratory study. Complement Ther Clin Pract. 2017;27:52-6.

5. Harris P, Rees R. The prevalence of complementary and alternative medicine use among the general population: a systematic review of the literature. Complement Ther Med. 2000;8(2):88-96.

6. Howard J. Do Bach flower remedies have a role to play in pain control?: A critical analysis investigating therapeutic value beyond the placebo effect, and the potential of Bach flower remedies as a psychological method of pain relief. Complement Ther Clin Pract. 2007;13(3):174-83.

7. Brasil. Ministério da Saúde. Gabinete do Ministro. Portaria $\mathrm{n}^{\circ}$ 971, de 3 maio de 2006. Aprova a Política Nacional de Práticas Integrativas e Complementares (PNPIC) no Sistema Único de Saúde. Diário Oficial da União, Brasília, DF. 2006;(84):20-25. Seção 1. [Acesso em 21 set. 2018]. Disponível em: http://bvsms.saude.gov.br/ bvs/saudelegis/gm/2006/prt0971_03_05_2006.html.

8. CFO. Conselho Federal de Odontologia. Decisão $\mathrm{n}^{\mathrm{o}} 451^{\circ}$ de outubro de 2008. [Internet] acesso em 21 de setembro de 2018. Disponível em: http://www.ihb.org.br/br/docs/desicao_cfo.pdf

9. CFO: Conselho Federal de Odontologia. [Internet]. Citado em 13 de Julho de 2018. Disponível em: http://cfo.org.br/

10.Facioli F, Soares AL, Nicolau RA. Terapia floral na Odontologia no controle de medo e ansiedade revisão de literatura. Em XIV Encontro Latino Americano de Iniciação Científica e X Encontro Latino Americano de Pós-Graduação- 
Universidade do Vale do Paraíba, 2010. Disponível em: http://www.inicepg.univap.br/cd/ INIC_2010/anais/arquivos/RE_0766_0632_01.pdf 11. Giorgio MA et al. Terapêuticas: alternativas para profissão. Rev ABO. 1994;2(4):234-41.

12.Fernandes Neto JA, Silva AMT, de Oliveira CL, Catão MHCV. Habilitação em laserterapia para cirurgiões-dentistas: uma análise por estados e regiões brasileiras. Arch Health Invest. 2017; 6(1):24-7.

13.Fernandes Neto JA, da Silva MGB, Simões TMS, Andrade FA, Batista ALA, Catão MHCV. Habilitação em acupuntura para cirurgiõesdentistas no Brasil: uma análise por estados e regiões. Arch Health Invest. 2017;6(4):181-84.

14. Gonçalves RN, Gonçalves JRSN, Buffon MCM, Negrelle RRB; de Albuquerque GSC. Práticas Integrativas e Complementares: inserção no contexto do ensino Odontológico. Rev ABENO. 2018;2:114-23.

\section{CONFLITO DE INTERESSES}

Os autores declaram não haver conflitos de interesse.

\section{AUTOR PARA CORRESPONDENCIA}

Maria Helena Chaves de Vasconcelos Catão mhelenact@zipmail.com.br

Submetido em 19/11/2018

Aceito em 12/03/2019 